\title{
La enseñanza de un uso de Se como \\ modificador pragmático
}

\section{Araceli Álvarez Cederborg María Reyes}

\section{Introducción}

La enseñanza de los usos de se constituye uno de los retos más difíciles para el profesor de español como segunda lengua, dado que esta forma realiza diversas funciones en el habla y que en la interacción cotidiana este pronombre se usa con mucha frecuencia.

En este trabajo nos ocupamos de un caso particular de se cuya aparición junto al verbo no modifica el significado semántico del mismo sino que señala una modificación en el nivel pragmático. Presentamos también una propuesta didáctica para su identificación en el habla cotidiana dirigida a estudiantes de nivel avanzado.

\section{Objetivo}

El objetivo de este trabajo es mostrar la identificación de un uso pragmático de se en diferentes intercambios conversacionales y ofrecer una propuesta didáctica para su trabajo en el aula.

\section{Justificación}

$\checkmark$

La claridad y el conocimiento que tengamos de nuestra propia lengua va a incidir directamente en el qué y cómo la enseñamos; de la misma manera que para reconocemos culturalmente. Determinados hábitos comunicativos reflejan ciertas prácticas sociales -un ejemplo concreto es el uso de se con alcance pragmático-. Identificar al menos uno de éstos puede representar un pequeño avance en la enseñanza del español a hablantes no nativos.

\section{Marco teórico}

4.1. El pronombre se como <reflexivo dativo $>$ e $<$ intensificador subjetivo $>$.

Como es sabido, el pronombre se realiza diversas funciones en español. Una de ellas, ha sido identificada y clasificada por Gómez Torrego (1992:11-16) como <se reflexivo dativo $>$. Este autor distingue dos tipos de dativo:

1.El pronombre como elemento no necesario (dativo expletivo):

* Juan se comió todo el pastel

* Juan no se cree todo lo que has dicho

* Juan se lo jugó todo en la lotería

* Juan no se sabe bien la lección

2. El pronombre como elemento necesario

* Juan se gana la vida como puede

* Los toreros se juegan la vida delante del toro

* Juan se saltó un semáforo ('se pasó el alto" en la norma mexicana) 11 Juan se llevó (se trajo) el dinero * Él se lo pierde

* Juan se las arregla como puede

Sobre este segundo tipo de construcciones, Gómez Torrego hace notar que la forma se no deja de ser pronombre pues comparte el paradigma reflexivo átono (me, te, nos), pero 
señala que no es con mutable por le, por lo que no podría considerarse una variante del complemento indirecto < dativo de interés o ético>, sino como una función autónoma. Señala que estas oraciones con dativo exigen además un complemento directo, por lo que siempre son transitivas y que, por otro lado, se suele decir que el pronombre en función de dativo es siempre expletivo, y como puede verse en los ejemplos que maneja, en algunos casos lo es, pero en otros no.

En la lista de ejemplos que ofrece aparecen casos como el de <arreglárselas> en los que se podría hablar de verbos doblemente pronominales. En otros ejemplos, como el de $<$ Juan se gana la vida...> podría hablarse de verbos <semipronominales>, ya que el pronombre resulta necesario para el verbo, y esto ocurre, sobre todo cuando el verbo muestra un uso figurado, o cuando se trata de frases más o menos fijas (semilocuciones) como $<$ ganarse la vida $>$, <jugarse la vida $>$, y lo sería también <pasarse el alto>. Sin embargo, dado que Gómez Torrego desea presentar una clasificación general de los valores gramaticales de se, no particuliza en el efecto que produce la presencia de este pronombre en casos como <comerse>, <saberse $>$, etcétera.

Si tratamos de analizar el valor que añade al verbo la presencia de se en verbos como $<$ comer>, <tomar>, < saber> etc., encontramos que el pronombre no cambia el significado lexical del verbo, pero le añade cierta significación adicional, que habría que identificar en cada caso.

En un trabajo sobre análisis de errores, Sonsoles Fernández (1977) trabaja con una clasificación de usos de se en la que incluye una función denominada <intensificador subjeti- vo> 1. Si bien no da ejemplos, la autora se refiere a un uso ${ }^{1}$ de este pronombre en el que su presencia tendría la función de "ponderar el objeto de la acción o intensificar la calidad o magnitud de la misma acción" (:117) Consideramos que los casos de se citados por Gómez Torrego corresponderían a esa función, ya que si analizamos el significado de los casos de dativo presentados por este autor y otros ejemplos que incluimos, pensamos que en ellos la presencia del pronombre intensifica la magnitud o calidad de la acción. Sin embargo, habría que distinguir dos subgrupos.

\section{Subgrupo A.}

$S e$ indica que el sujeto que realiza la acción goza o aprovecha de ésta y se cuantifica la porción del objeto que fue aprovechado. En otros casos el énfasis se pone en el esfuerzo que significó la realización de la acción.

Ejemplos:

* Juan se comió todo el pastel

* Luis se tomó mi cerveza.

* Juan y Luis se desayunaron unos chilaquiles muy ricos.

* Ayer Luis se fumó sólo tres cigarros.

* José se leyó todo el artículo mientras esperaba al doctor.

* Ellos se conocen muy bien la ciudad.

* José se sabe muy bien la lección, creeme.

* El niño se aprendió las tablas de multiplicar muy rápido.

* ¿Se memorizaron ustedes todas las reglas?

* José se ganó el primer premio en el concurso.

\section{Subgrupo B.}

Con verbos de movimiento se enfatiza que el sujeto se desplaza de un lugar a otro portando consigo un elemento ( por lo regular un objeto o una persona) que se encontraba en

'Esta clasificación fue tomada por la autora de F. Marsá (1984) Cuestiones de sintaxis española. Barcelona: Ariel. 
el misma lugar o entorno que el sujeto cuando se inició el movimiento.

Es decir, se lexicaliza el lugar de ubicación del elemento.

Ejemplos:

* Juan se llevó mi libro a su casa.

Compárese esta oración con Juan llevó mi libro a su casa. En el primer enunciado entendemos que el libro estaba en el entorno no que rodeaba a Juan cuando se realizó el movimiento. En el segundo, no se lexicaliza la ubicación del libro.

* Luis se trajo unas tortas.

(Compárese con Luis trajo unas tortas). En el primer enunciado sabemos que las tortas proceden del lugar en donde estaba Luis al realizar el desplazamiento; en el segundo, lo ignoramos, pudo haberlas traído de la tienda.

* La próxima vez que venga arriba, súbase los libros. (Se entiende que los libros están con él). Juan se bajó los libros para que los veamos. (Se entiende que los libros estaban arriba, con Juan).

No incluimos en estos dos subgrupos algunos casos de dativo considerados por Gómez Torrego, ya que, ejemplos como <jugárselo todo en la lotería $>$, <jugarse la vida $>$, $<$ ganarse la vida>, <pasarse un alto >, <perderse (de) algo $>\mathrm{o}<$ arreglárselas $>$, son claros casos de locuciones o semi locuciones verbales en los que el verbo acompañado del pronombre adquiere una significación que es diferente de la que tiene el verbo en ausencia del pronombre. Los casos de <creerse $>$ e $<$ imaginarse $>$ preferimos dejarlos de lado por ahora, ya que pensamos que según el contexto pueden cambiar su significado. Por ejemplo, entre <Juan se cree todo lo que le dices $>$ y $<$ Juan cree todo lo que le dices $>$ no parece haber un cambio en el significado lexical del verbo. Pero cuando decimos $<$ Juan se cree muy listo>, ahi se hace evidente que hablamos de otro verbo <creerse $>$ cuyo significado ya no es $<$ creer $>$ (tener por cierta una cosa) sino <tenerse en una opinión $>$. En cuanto a <imaginar $>$ e $<$ imaginarse $>$, encontramos también ciertas diferencias en el significado lexical de uno y otro verbos. En la frase $<\mathrm{El}$ autor imaginó un mundo en el que luchaban fuerzas antagónicas el verbo <imaginar> es representar creativamente una cosa en la mente, crearla en la imaginación. En cambio, en la oración <Juan se imagina que yo tengo mucho dinero> $<$ imaginarse $>$ se emplea más bién con el sentido de < suponer $>$, < pensar $>$.

Incluimos, en cambio, una serie de verbos que no menciona Gómez Torrego que guardan una gran similitud con verbos como $<$ comerse $>$.

Como puede observarse los dos subgrupos de verbos A y B coinciden en lo siguiente:

1.Son transitivos y se acompañan de una frase de objeto directo obligatoria.

2. Tienen una contraparte igualmente transitiva no pronominal (Ayer leí un artículo / ayer me leí un artículo).

3. Tienen el mismo significado lexical que su contraparte no pronominal, aunque es notorio que la presencia del pronombre aporta cierta información sobre la calidad o magnitud de la acción, es decir, se produce un ligero cambio en la semántica del verbo.

4.El agente real de la accción tiene el rasgo [+animado].

4.2. El pronombre se en el nivel pragmático la conversación informal

Tomamos como referencia teórica la propuesta de Briz G.(2000), sobre cómo se comenta un texto coloquial. En él, el autor define la 
conversación como un tipo de discurso oral cuyas características son:

$>$ Inmediatez comunicativa: aquí ahora ante ti.

$>$-Dinamismo: cambio de papel de los interlocutores, de hablante a oyente y viceversa.

$>$ Carácter cooperativo: ambos interlocutores colaboran.

$>$ Alternanacia de turno: no predeterminada.

Los primeros tres rasgos son compartidos por otros dicursos orales, como el debate y la entrevista. Sin embargo, el rasgo que la distingue de estos discursos es la alternanacia de turnos no predeterminada (:51).

El discurso conversacional tiene a su vez características propias que nos llevan a dividirlo en dos tipos de conversaciones prototípicas: la conversación informal y la formal, las cuales se pueden distinguir por los siguientes rasgos:

Los ejemplos que mostramos y con los que trabajamos nuestro análisis pertenecen a la conversación informal o coloquial.

\begin{tabular}{|l|c|c|}
\hline Rasgos & Conversación informal & Conversación formal \\
\hline $\begin{array}{l}\text { Relación de } \\
\text { proximidad } \\
\text { entre los } \\
\text { hablantes }\end{array}$ & + & - \\
\hline Cotidianidad & + & - \\
\hline Planificación & + & - \\
\hline Finalidad & Interpersonal & Transaccional \\
\hline
\end{tabular}

Una vez aclarado lo anterior, es necesario hacer también una caracterización de la conversación como nivel de análisis lingüístico. Briz G. (:52) nos dice que el estudio de la actuación lingüística puede comprender tres niveles: el de la enunciación se refiere a las acciones de los actos de habla; el de la argumentación son "las intenciones y valoraciones implícitas o explícitas de esos actos de habla" (:53), y el de la interacción o conversación es el de las relaciones con el otro.

Para cada uno de estos niveles el autor propone sus respectivas unidades de análisis, de tal manera que se forma el siguiente cuadro:

\begin{tabular}{|l|c|c|}
\hline $\begin{array}{l}\text { Nivel de análisis } \\
\text { lingüístico }\end{array}$ & Unidad de Análisis & Equivale a: \\
\hline 1. Enunciación & $\begin{array}{c}\text { Acto de habla o } \\
\text { enunciado }\end{array}$ & Hacer \\
\hline 2. Argumentación & Intervención & Actuar \\
\hline 3. Interacción & Intercambio & Interactuar \\
\hline
\end{tabular}


De la suma de intercambios resulta la conversación, que es la unidad de análisis interaccional.

Nuestro análisis se centra en el nivel de la interacción cuya unidad de análisis se forma con dos intervenciones sucesivas, una de inicio y una de reacción (intercambio). Es necesario, entonces, aclarar que la intervención es "un enunciado o conjunto de enunciados emitidos por un interlocutor de forma continua o discontinua y vinculados por una estrategia única de acción e intención" (53). Las intervenciones pueden ser de inicio: preguntas, juicios, invitaciones, reproches, peticiones, etc., o de reacción, como: respuestas, conformidades, aceptaciones, excusas, concesiones, valoraciones, acuerdo, desacuerdo, etcétera.

En resumen, el intercambio tiene como constituyente inmediato la intervención (de inicio o de reacción) y este último, a su vez, el de acto de habla.

\section{Análisis}

Además del grupo de verbos que pueden acompañarse de se sin cambiar fundamentalmente el significado lexical del verbo, hemos identificado otro grupo, que no incluye Gómez Torrego, en el que la presencia del pronombre parecería aportar más bien una rasgo o modificación de orden pragmático, no semántico como en los dos grupos analizados. A esta función de se la denominamos $<$ modificador pragmático $>$.

$\mathrm{Al}$ iniciar este trabajo pensamos que este otro grupo podría ser incluido como un tercer subgrupo de ejemplos de se como intensificador subjetivo, sin embargo, después de analizar diferentes ejemplos encontramos que no es posible, que tendríamos que hablar de dos grupos:
1. Uno (subgrupos A y B) en el que el pronombre cumple la función de intensificador subjetivo, es decir, intensifica la magnitud o calidad de la acción, y

2. Otro, en el que la presencia del pronombre marca una diferencia en el nivel pragmatico. Dentro de este grupo presentamos los siguientes ejemplos de intercambios conversacionales:

(1)

A: Tengo mucha sed, tú, no?

B: Sí, yo también. Sácate unos refrescos del refri, ¿no?

(2)

A: Está muy rico el café, ¿por qué no te prendes un cigarrito? B: Ah, órale.

(3)

A: Todavía no está lista la comida.

B: Oye, pues sírvete mientras una botanita, ¿no?

(4)

A: iQué frío está haciendo!

B: Ay sí, ¿Por qué no te preparas un cafecito caliente? Se me antoja uno.

(5)

A: Oye, como que estamos muy callados, ¿no?

B: ¿Por qué no te pones una musiquita y platicamos?

Estos ejemplos son intercambios con una intervención de cada uno de los interlocutores, en los que se presenta el uso de se, tanto en intervenciones de inicio (ejemplo 2), como de reacción (ejemplos 1, 3, 4, 5).

El uso de pronombre se, expresa la intención del hablante de que el resultado de la acción de su petición por parte de su interlocutor sea para compartir entre ambos hablantes -quizá más-. Respecto de cada uno de los ejemplos podemos decir que:

Ejemplo 1: el resultado de sacar un refresco 
del refrigerador va a beneficiar a los dos por que ambos tienen sed, y van a compartir el líquido.

Ejemplo 2: "Prenderte un cigarro" quiere decir uno tú y uno yo, vamos a fumar, vamos a compartir.

Ejemplo 3: "sírvete mientras una botanita" no es sólo para ti, es: yo te pido que sirvas una botana para que comamos algo mientras está la comida, para compartirla.

Ejemplo 4. "por que no te preparas un cafecito" es: vamos a tomar juntos algo para calentamos, vamos a compartir, tú tienes frío, a mí se me antoja uno.

Ejemplo 5. "por qué no te pones..." es: pon una música que los dos podamos oír y que nos guste, vamos a compartirla y a platicar.

De esta manera identificamos que este uso particular de se, se debe a que el hablante tiene la intención de que el resultado de la acción sea compartible por el hablante y el interlocutor, por lo menos. Por esta razón habría que aclarar que hay un limitado inventario de verbos que aceptan la presencia del pronombre se con ésta función, y que estos verbos en otro contexto aceptan también la presencia del pronombre se con la función de objeto indirecto, particularidad que puede ser motivo de conflicto lingüístico en un momento dado para el no hispanohablante.

Aunado a la presencia de se como modificador pragmático localizamos en los mismos turnos de habla usos también pragmáticos de:

* Diminutivos: cafecito, musiquita, botanita.

* La partícula ¿no? en función fática $(1,3)$

* La partícula "oye" como indicador de la proximidad entre los hablantes(3)

* La forma interrogativa $(2,4,5)$ en oposición al uso del imperativo $(1,3)$

* Elementos léxicos "pues >pus>pos"

* Es frecuente que el verbo se conjugue en imperativo o presente con valor de impe rativo.

\section{Propuesta didáctica}

Como puede verse, el uso del pronombre se en el tipo de oraciones analizadas podría ser motivo de conflicto lingüístico en las situaciones de vida cotidiana que enfrenta el estudiante de español inmerso en la cultura mexicana -pensamos en los estudiantes del CEPE-, ya que el estudiante podría confundir fácilmente este uso de se como modificador pragmático con el caso de pronombre se como objeto indirecto. Con objeto de hacer consciente al estudiante de español de este posible foco de conflicto lingüístico, elaboramos un ejercicio electrónico encaminado a que el estudiante logre identicar el uso del pronombre se como modificador pragmático, distinguiéndolo del uso de se como pronombre de objeto indirecto y del mensaje que se comunica cuando no está presente.

Escogimos el medio electrónico ya que nos permite contextualizar los enunciados mediante el video, cuyas ventajas pedagógicas son bien conocidas.

El ejercicio que presentamos constituye únicamente una muestra del uso pragmático que nos ocupa. Habría que trabajar más en el diseño de otro tipo de ejercicios ya que solamente presentamos uno dirigido a la identificación de este uso especial de se, no a su producción oral. Por otra parte habría que señalar que está orientado a estudiantes de nivel avanzado.

En cuanto a las muestras de habla que se ofrecen cabría añadir que fueron retomadas de interacciones reales recopiladas por las autoras a lo largo del tiempo y que fueron recreadas en el video por dos voluntarias. 
Descripción del ejercicio.

Se presentan tres minivideos de conversación informal entre dos interlocutores. En uno de ellos el pronombre se emplea como modificador pragmático; en otro con valor de objeto indirecto y en el tercero no se emplea el pronombre. En las tres situaciones se emplea el verbo prender. El alumno ve y escucha los tres videos y posteriormente relaciona cada video con un enunciado en el que se explica la intención que tuvo el hablante al emplear o no, el pronombre se (en su variante te). Los intercambios conversacionales son los siguientes:

\section{INTERCAMBIO 1}

A: Está muy rico el café, préndete un cigarrito, ¿no? Se me antojó fumar. B: Ah, órale. (Le ofrece el cigarro y prenden ambas un cigarro cada una).

\section{INTERCAMBIO 2}

A: Ay, cómo tarda Juan, tengo ganas de fumar.

B: Pues préndete un cigarro mientras llega. Total, cuando llegue lo apagas y ya.

\section{INTERCAMBIO 3}

A: Ay, jcuántos moscos!

B: Prende un cigarro, el humo los espanta.

INSTRUCCIÓN PARA EL ESTUDIANTE: Relaciona cada video con una de las opciones que se ofrecen.

La función del pronombre "te" en la intención del hablante es que el interlocutor pren-

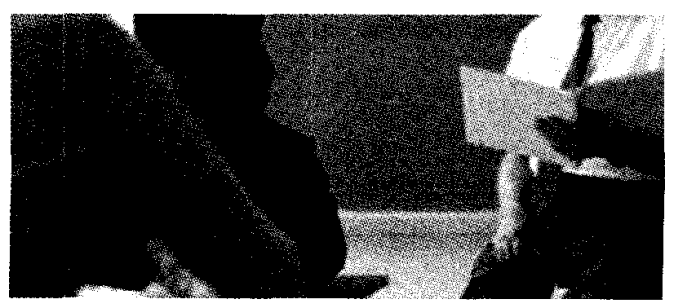

da un cigarro :

Para él mismo.

Para ambos interlocutores

Con algún otro propósito.

\section{Comentarios finales}

Como hemos visto, el pronombre se realiza diversas funciones, no sólo sintácticas y semánticas sino también pragmáticas. En este trabajo hemos identificado una función pragmática de se en el español mexicano, no del todo ajena a otras normas hispanoamericanas. Sin embargo, es un tema que no se ha trabajado en el campo de la enseñanza del español a no hispanohablantes. Esperamos que la identificación de este uso permita a profesores y estudiantes una mejor comprensión de la lengua oral y se facilite con ello la enseñanza y el aprendizaje de este uso.

\section{Ejercicio}

En grupos de tres personas elegir por lo menos uno de los verbos del cuadro y pensar en tres posibles intercambios orales contextualizados en los que la función de se en la intención del hablante sea la de realizar una acción...
a. para él mismo
b. para ambos interlocutores
c. con algún otro propósito calentar(se) comprar(se) hacer(se) la-var(se) servir(se)

Bibliografía:

BRIZ, Gómez, A. y Grupo VaI.Es.Co. 2000. ¿Cómo se comenta un texto coloquial?,

Barcelona, Ariel.

FERNÁNDEZ, Sonsoles. Interlengua y análisis de errores en el aprendizaje del español como lengua extranjera. Madrid, 1977 EDELSA. GÓMEZ, Torrego, Leonardo Valores gramaticales de Se, Cuadernos de lengua española. Madrid: Arco Libros, S. A., 1992. 
